\title{
Alívio da dor após bloqueio do gânglio ímpar e ablação por radiofrequência em pacientes com coccigodínia: Uma revisão sistemática*
}

\section{Improvement in Pain Following Ganglion Impar Blocks and Radiofrequency Ablation in Coccygodynia Patients: A Systematic Review}

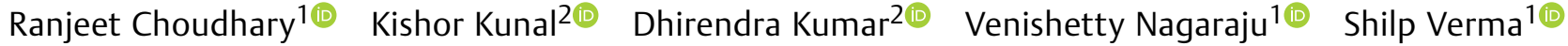 \\ ${ }^{1}$ Department of Orthopaedics, Instituto de Ciências Médicas da Índia \\ (AlIMS), Raipur, Chhattisgarh, Índia \\ 2 Department of Orthopaedics, Instituto de Ciências Médicas da Índia \\ Endereço para correspondência Kishor Kunal, MS (Orthopaedics), \\ Dept. of Orthopaedics, AlIMS, Jodhpur, PIN 342005, Rajasthan, Índia \\ (e-mail: drkunal2408@gmail.com).
} (AIIMS), Jodhpur, Rajasthan, Índia

Rev Bras Ortop 2021;56(5):558-566.

\section{Resumo \\ Palavras-chave \\ - cóccix/diagnóstico por imagem \\ - cóccix/lesões \\ - cóccix/fisiopatologia \\ - ablação por radiofrequência}

Quase $90 \%$ dos casos de coccigodínia podem ser tratados por meio de tratamento clínico conservador; os $10 \%$ restantes precisam de outras modalidades invasivas para o alívio da dor, como o bloqueio do gânglio ímpar (BGI) ou ablação por radiofrequência (ARF) do gânglio ímpar. Com o objetivo de avaliar a eficácia do BGl e ARF do gânglio ímpar no controle da dor em pacientes com coccigodínia, foi realizada uma pesquisa sistemática no PubMed, MEDLINE e Google Scholar, a fim de identificar estudos que relatam o alívio da dor, em termos de Escala Visual Analógica (EVA) ou dos seus homólogos, após o BGl ou ARF em pacientes com coccigodínia por 2 autores diferentes, de acordo com as diretrizes PRISMA. Foram definidos sete estudos com um total de 189 pacientes (104 no grupo BGl e 85 no grupo ARF). No grupo BGI, a média da pontuação EVA melhorou de 7,83 no início do estudo para 3,11 no acompanhamento de curto prazo, 3,55 no acompanhamento de médio prazo e 4,71 no acompanhamento de longo prazo. No grupo ARF, a média da pontuação EVA melhorou de 6,92 no início do estudo, 4,25 no acompanhamento de curto prazo e 4,04 no acompanhamento de longo prazo. No grupo BGI foram relatadas $13,92 \%$ de falhas (11/79) e complicações de $2,88 \%$ (3/104), enquanto que no grupo ARF foram relatadas $14,08 \%$ de falhas (10/71) e nenhuma complicação $(0 \%)$. A taxa total de êxito foi $>85 \%$ em qualquer uma das modalidades. O BGI e ARF do gânglio ímpar fornecem um método

Trabalho desenvolvido no Instituto de Ciências Médicas da Índia (AIIMS), Raipur, Índia.

recebido

27 de Dezembro de 2020

aceito

07 de Abril de 2021
DOI https://doi.org/

$10.1055 / \mathrm{s}-0041-1735829$. ISSN $0102-3616$
(C) 2021. Sociedade Brasileira de Ortopedia e Traumatologia. All rights reserved.

This is an open access article published by Thieme under the terms of the Creative Commons Attribution-NonDerivative-NonCommercial-License, permitting copying and reproduction so long as the original work is given appropriate credit. Contents may not be used for commercial purposes, or adapted, remixed, transformed or built upon. (https://creativecommons.org/ licenses/by-nc-nd/4.0/)

Thieme Revinter Publicações Ltda., Rua do Matoso 170, Rio de Janeiro, RJ, CEP 20270-135, Brazil 


\author{
Abstract \\ Keywords \\ - Coccyx/diagnostic \\ imaging \\ - Coccyx/injuries \\ - Coccyx/ \\ physiopathology \\ - Radiofrequency \\ ablation
}

confiável e provavelmente excelente no controle da dor, em pacientes com coccigodínia que não respondem ao tratamento médico conservador. No entanto, deve ser estabelecido um limite entre os que responderam, os que não responderam e aqueles não respondedores tardios, sendo necessários estudos mais amplos com acompanhamento mais longo ( $>1$ ano).

Nearly $90 \%$ of cases of coccygiodynia can be managed with conservative medical treatment; the remaining $10 \%$ need other invasive modalities for pain relief such as GanglionImpar block (GIB) or Radiofrequency ablation (RFA) of Ganglionlmpar. With a purpose to determine the efficacy of GIB and RFA of Ganglion Impar in controlling pain in coccygiodynia patients a systematic research was conducted of PubMed, MEDLINE and Google Scholar to identify studies reporting the pain relief in terms of Visual Analogue Scale (VAS) or its counterparts following GIB or RFA in coccygiodynia patients by 2 different authors according to PRISMA guidelines. 7 studies were delineated with a total of 189 patients (104 in GIB group and 85 in RFA group). In GIB group, mean VAS improved from 7.83 at baseline to 3.11 at short term follow-up, 3.55 at intermediate term follow-up and 4.71 at long-term follow-up. In RFA group, mean VAS improved from 6.92 at baseline, 4.25 at short term follow-up, 4.04 at long-term follow-up. In GIB group, $13.92 \%$ failure $(11 / 79)$ and $2.88 \%$ complication $(3 / 104)$ were reported while in RFA group, $14.08 \%$ failure (10/71) and no complications (0\%) were reported. Total success rate was $>85 \%$, with either of modalities. GIB and RFA of Ganglionlmpar provides a reliable and probably an excellent method of pain control in coccygiodynia patients not responding to conservative medical treatment. However, a demarcation between responders, non-responders and late non-responders should be considered and larger studies with longer follow-up (> 1 year) are needed.

\section{Introdução}

Em 1859, Simpson introduziu o termo Coccidínia, referindose a dor e a sensibilidade ao redor da região sacrococcígea. ${ }^{1} \mathrm{~A}$ dor geralmente piora ao permanecer sentado por muito tempo em superfícies duras, estreitas ou desconfortáveis e ao levantar da posição sentada. ${ }^{2,3} \mathrm{~A}$ coccidínia possui uma natureza multifatorial, provavelmente de origem idiopática. ${ }^{2}$ A etiologia traumática é a mais comumente observada, sendo que os casos podem se manifestar de várias formas, como luxação posterior, hipermobilidade e espículas do cóccix. A infecção e os tumores do cóccix podem ser consideradas causas raras. ${ }^{2,4} \mathrm{~A}$ obesidade e o gênero feminino estão associados a um maior risco de desenvolver coccidínia. A incidência é cinco vezes maior em mulheres do que em homens. ${ }^{2}$ Além disso, adolescentes e adultos são comumente afetados em comparação com as crianças. 2,5

A maioria dos casos de coccigodínia pode ser tratada de forma conservadora, como os fármacos anti-inflamatórios não esteroides (AINEs), modificação do estilo de sentar, uso de almofadas coccígeas, reabilitação do assoalho pélvico, terapia por eletroestimulação nervosa transcutânea (ENT), terapia por ondas de choque extracorpórea (ESWT) e fisioterapia com até $90 \%$ de taxa de resolução. ${ }^{6-9}$

Os poucos casos que não são resolvidos com o tratamento conservador mencionado acima, necessitam de uma intervenção invasiva, ou seja. intervenções cirúrgicas e não cirúrgicas.
São mencionadas na literatura várias intervenções cirúrgicas e não cirúrgicas. As modalidades de tratamento intervencionista não cirúrgico, como a injeção peridural caudal de esteroide, o bloqueio ganglionar ímpar (BGI), ablação por radiofrequência e a neurólise química do gânglio ímpar podem ser usadas em pacientes refratários. A intervenção cirúrgica consiste em coccigectomia, mas raramente é necessária, sendo usada somente como último recurso. ${ }^{3,7,8,10}$

O gânglio ímpar é um gânglio retroperitoneal solitário, representando a terminação fusionada das cadeias simpáticas paravertebrais bilaterais, localizadas ao nível do cóccix. É a estação de retransmissão sensorial do estímulo nociceptivo da zona pélvica e fibular. O bloqueio do gânglio ímpar foi empregado para tratar a dor oncológica perineal (reto, vulva, próstata), bem como a dor crônica de origem não oncológica, isto é, a coccigodínia, síndrome da dor pélvica crônica e etc. O BGI pode ser realizado utilizando diversas modalidades, como os anestésicos locais, corticosteroides, clonidina, toxina botulínica, álcool, ablação por radiofrequência ou a crioablação. ${ }^{11,12}$

A injeção de esteroides isolada ou acompanhada da terapia de lesão por radiofrequência (termocoagulação por radiofrequência, radiofrequência pulsátil) e a terapia do gânglio ímpar são comumente usadas na coccidínia recalcitrante. ${ }^{7,13-15}$ No entanto, apenas alguns estudos avaliaram a eficácia em longo prazo deste procedimento de injeção, não estando disponível nenhum ensaio comparativo randomizado. O objetivo da presente revisão sistêmica é rastrear a literatura 
sobre a eficácia do bloqueio do gânglio ímpar, avaliando os efeitos a longo prazo da desnervação do gânglio ímpar.

\section{Materiais e Métodos}

\section{Objetivos}

1. Estudar a melhora na intensidade da dor na escala EVA, após o bloqueio do gânglio ímpar em pacientes com coccigodínia

2. Estudar a melhora na intensidade da dor na escala EVA, após a ablação por radiofrequência do gânglio ímpar em pacientes com coccigodínia

3. Estudar a diferença na melhora da dor na escala EVA, após o bloqueio do gânglio ímpar e ablação por radiofrequência do gânglio ímpar em pacientes com coccigodinia

\section{Metodologia}

Uma busca abrangente e estruturada foi realizada usando o banco de dados da Cochrane Library, Medline, Embase e a base de dados de revisões sistemáticas Cochrane (CDSR). A estratégia de busca usada para identificar os estudos relevantes foi baseada no modelo PICO: população, intervenção, comparação e medidas de resultados. Os termos de pesquisa da população incluídos foram coccidínia, dor no cóccix, coccigodínia crônica, recalcitrante ou mais de 3 meses. Os termos de pesquisa da intervenção incluídos foram bloqueio do gânglio ímpar, ablação por radiofrequência do bloqueio pré-sacral ou radiofrequência pulsátil. Nenhum termo de pesquisa foi usado no grupo de comparação. Para o grupo de resultados, os termos de pesquisa consistiram em melhora da dor, pontuação EVA, pontuação EAN e o estudo deve ter pelo menos 6 meses de acompanhamento. Os termos da pesquisa população, intervenção e resultados foram combinados com “OU.” Os termos intergrupos foram combinados usando o termo de pesquisa "E." As citações foram armazenadas e organizadas.

Critérios de inclusão e exclusão: Os estudos para inclusão deveriam atender aos seguintes critérios: (1) estudo com grupo etário >18 anos (2) presença de sintomas por no mínimo 3 meses de duração (3) participantes que não tenham apresentado alívio da dor após o tratamento conservador (4) acompanhamento de pelo menos 6 meses. Seriam excluídos do estudo, caso apresentassem um dos seguintes critérios: (1) participantes que tenham sido submetidos a qualquer tipo de injeção local na região coccígea; (2) estudos que descrevem intervenções cirúrgicas, envolvendo a coluna lombar ou a pelve, incluindo os pacientes com câncer e/ou cistos, (3) relatos de casos, apresentações em conferências e ensaios não publicados.

Foi utilizado os critérios PRISMA (Itens Preferidos de Relatório para uma revisão sistemática e meta-análise) para rastrear os resultados da pesquisa e selecionar os artigos para inclusão na revisão. Dois revisores (RC e KK) selecionaram e analisaram independentemente os resultados da pesquisa. A primeira etapa do processo de seleção envolveu a identificação de todos os estudos possíveis e relevantes nas bases de dados citadas. Estes foram selecionados, analisando os títulos, bem como os resumos. Após a conclusão desta etapa, foram destacados os estudos mais relevantes para o processo de identifi- cação posterior. Isso envolveu a recuperação de textos completos dos artigos, sendo posteriormente comparados com os critérios de inclusão e exclusão estabelecidos. As citações duplicadas, assim como os estudos que não correspondiam aos nossos parâmetros revisionais, foram retirados e tomada uma decisão final quanto à seleção do artigo. As discordâncias foram resolvidas por um revisor extra, através de discussão e consenso com os dois revisores principais.

A avaliação da força metodológica e da validade dos estudos incluídos (avaliação do risco de viés) foi realizada com a utilização de uma estrutura para garantir a reprodutibilidade do processo. A estrutura usada foi a ferramenta de avaliação de qualidade do NIH para estudos antes-depois (pré-pós). Usando a ferramenta do $\mathrm{NIH}$, os revisores analisaram, avaliaram e classificaram os estudos independentemente em três categorias - "Bom," "Regular" e "Deficiente." o décimo segundo parâmetro do questionário (grupo intervenção) não se aplica a nenhum dos nossos estudos, portanto, apenas onze itens foram usados para ratificar a qualidade do estudo. Caso fossem assinalados 9 ou mais itens no questionário como "Sim," o estudo seria classificado como "Bom," se 6-8 perguntas fossem marcadas como "Sim," então seria classificado como "Regular" e se apenas 5 itens ou menos apresentassem resposta "Sim," então a qualidade do estudo seria descrita como "Deficiente."

Extração de dados: Os dados demográficos e epidemiológicos dos estudos incluídos na revisão foram tabulados em planilha do Microsoft Excel (-Tabelas 1 e 2). Os parâmetros estudados incluíam o número de pacientes em cada estudo, história de trauma, idade média, IMC, abordagem de bloqueio ganglionar, material de injeção/ técnica de ablação e sistema de pontuação utilizado. Conforme o objetivo da revisão no desfecho primário, os escores de dor foram avaliados com escala visual analógica numérica (EVA) e a escala de avaliação numérica (EAN) na pré-injeção/pré-ablação e após o procedimento. De acordo com a disponibilidade dos dados, foram divididos em curto prazo (3-4 semanas), intermediário (3 meses) e longo prazo (6 meses). Entre as outras variáveis medidas estavam as complicações e as falhas (pacientes sem melhora ou pouca melhora quantificável de acordo com o autor do estudo em questão) no desfecho secundário.

As pontuações EVA e EAN foram utilizadas para avaliação do resultado primário. A pontuação EAN tem uma forte correlação positiva com a pontuação EVA, portanto, a pontuação EAN pode ser substituída pela EVA, para fins de avaliação da dor durante o acompanhamento. ${ }^{16} \mathrm{~A}$ análise dos dados envolveu o cálculo da média ponderada dos vários parâmetros demográficos. Embora não tenhamos sido capazes de realizar meta-análises, devido à heterogeneidade dos dados do estudo (analisados usando o teste estatístico $\mathrm{I}^{2}$ ), descrevemos os resultados estatísticos na forma de valores de $p$ e intervalos de confiança de 95\% (ICs), caso fossem relatados em qualquer estudo.

Processo de triagem: Um total de 50 citações foram identificadas após a pesquisa bibliográfica nos bancos de dados Cochrane Library, Medline, Embase e banco de dados Cochrane de revisões sistemáticas (CDSR). Após a primeira triagem, 21 estudos preencheram os critérios de inclusão. 
Tabela 1 Características dos vários estudos comparando o BGI no tratamento da coccigodínia

\begin{tabular}{|c|c|c|c|c|}
\hline & Gonnade et al & Sencan et al & Sir and Eksert & Sagir et al \\
\hline Ano & 2017 & 2018 & 2019 & 2020 \\
\hline Número & 31 & 28 & 25 & 20 \\
\hline Idade (Média, em anos) & 42,9 & - & 42,64 & - \\
\hline IMC (Médio, em kg/m²) & - & 29,49 & 24,73 & - \\
\hline $\mathrm{H} / \mathrm{d}$ trauma & 12 & - & - & 21 \\
\hline Abordagem & Sacrococcígea & Sacrococcígea & Sacrococcígea & $\begin{array}{l}\text { Sacrococcígea/ } \\
\text { Transcoccígea }\end{array}$ \\
\hline $\begin{array}{l}\text { Sistema (s) de } \\
\text { pontuação usado (s) }\end{array}$ & EAD, ODI & EVA, LANSS, SF-12 & EAND, LS & EVA \\
\hline Material & $\begin{array}{l}\text { 3-5 ml de Bupivacaína } \\
(0,5 \%)+1 \mathrm{ml} \\
\text { Metilprednisolona } \\
(40 \mathrm{mg})\end{array}$ & $\begin{array}{l}3 \mathrm{ml} \text { de Bupivacaína } \\
(0,5 \%)+1 \mathrm{ml} \\
\text { Metilprednisolona } \\
(40 \mathrm{mg})\end{array}$ & $\begin{array}{l}2 \mathrm{ml} \text { de Bupivacaína } \\
(0,25 \%)+1 \mathrm{ml} \\
\text { Triancinolona } \\
(40 \mathrm{mg})\end{array}$ & $\begin{array}{l}\text { Bupivacaína } \\
(0,25 \%)+1 \mathrm{ml} \\
\text { Metilprednisolona } \\
(40 \mathrm{mg}) \text { totalizando } 10 \mathrm{ml}\end{array}$ \\
\hline EVA- inicial & 7,9 & 7,89 & 8,0 & 7,4 \\
\hline EVA- curto prazo & 2,03 & 2,39 & 3,36 & 5,5 \\
\hline EVA- médio prazo & 2,48 & 3,11 & 4,04 & 5,2 \\
\hline EVA- longo prazo & 3,23 & 3,89 & 7,24 & 5 \\
\hline Sem melhora & 1 & 7 & - & 3 \\
\hline Complicação & 0 & $\begin{array}{l}2 \text { (reação vasovagal } \\
\text { secundária e } \\
\text { aumento } \\
\text { transitório da dor) }\end{array}$ & $\begin{array}{l}1 \text { (bradicardia e } \\
\text { hipotensão) }\end{array}$ & 0 \\
\hline
\end{tabular}

Tabela 2 Características dos vários estudos comparando ARF do gânglio ímpar no tratamento da coccigodínia

\begin{tabular}{|l|l|l|l|l|}
\hline & Demircay et al. & Gopal and McCroy & Adas et al. & Sir and Eksert \\
\hline Ano & 2010 & 2012 & 2016 & 2019 \\
\hline Número & 10 & 20 & 41 & 14 \\
\hline Idade (Média, em anos) & 49,2 & 51,3 & 46,68 & 42,52 \\
\hline IMC (Médio, em kg/m ${ }^{2}$ ) & - & - & 26,46 & 27,98 \\
\hline H/d trauma & 4 & 15 & 24 & - \\
\hline Abordagem & Transcoccígea (preferida) & Sacrococcígea & Transcoccígea & Sacrococcígea \\
\hline $\begin{array}{l}\text { Sistema(s) de pontuação } \\
\text { usado(s) }\end{array}$ & EVN & EVA & EVA & EAND, LS \\
\hline Método & $80 \mathrm{C} \times 120$ segundos & Pulsado @ 42C & 80 C x 90 segundos & $42 \mathrm{C} \times 120 \mathrm{~s} \times 3$ ciclos \\
\hline EVA- inicial & 8,7 & 6,82 & 6,22 & 7,85 \\
\hline EVA- curto prazo & 2,1 & 3,55 & 5,37 & 3,5 \\
\hline EVA- médio prazo & - & - & - & 3,14 \\
\hline EVA- longo prazo & 2,9 & 2,55 & 5,05 & 4,05 \\
\hline Sem melhora & 1 & 5 & 4 & - \\
\hline Complicação & - & 0 & & 0 \\
\hline
\end{tabular}

Estes então foram submetidos à segunda etapa do processo de triagem, em que os textos completos de todos os artigos foram cuidadosamente analisados.

Finalmente, 7 estudos foram selecionados para a revisão 4 sobre o bloqueio do gânglio ímpar e 4 na categoria de ablação por radiofrequência ( 1 artigo de Sir e Eksert era comum a ambos). Na - Fig. 1 é fornecido um fluxograma que descreve o processo de triagem e identificação, juntamente com os motivos de exclusão.

Dois estudos eram prospectivos, enquanto os cinco restantes eram estudos retrospectivos. Nenhum foi um ensaio clínico randomizado. Dois estudos avaliaram o efeito e a eficácia do bloqueio ganglionar ímpar, com o emprego de um agente anestésico local e um esteroide. Três estudos avaliaram o papel 


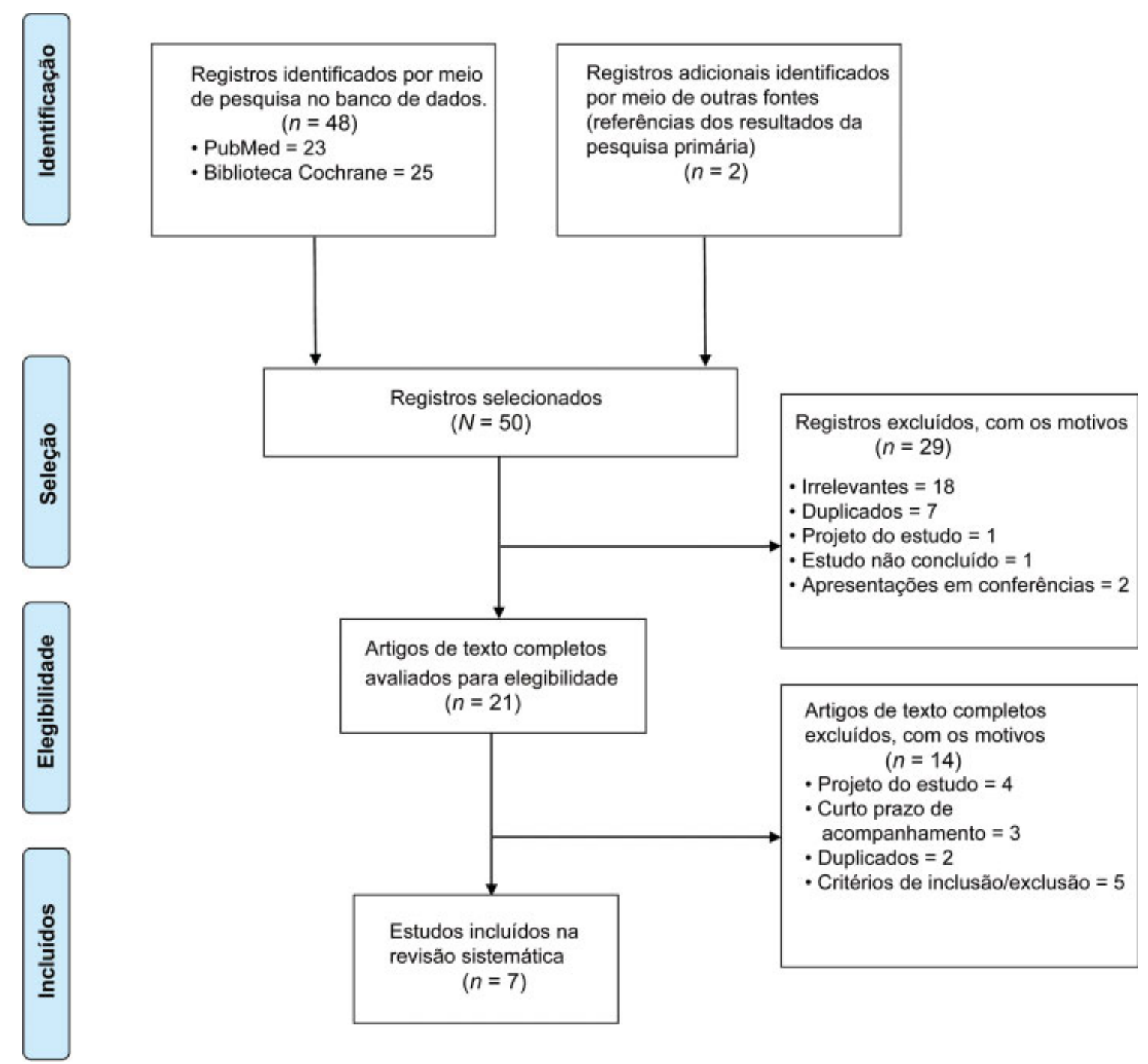

Fig. 1 Fluxograma das diretrizes PRISMA mostrando a inclusão e exclusão de estudos para revisão sistemática.

da ablação por radiofrequência convencional do gânglio ímpar. Um estudo comparou os dois grupos mencionados acima, enquanto outro comparou apenas o bloqueio e o bloqueio + ablação. Assim, neste último estudo, apenas os dados do primeiro grupo de pacientes foram utilizados para fins de revisão. Para fins de discussão, foram criados 2 grupos: o grupo BGI (recebendo apenas o bloqueio do gânglio ímpar) e o grupo ARF (recebendo apenas ablação por radiofrequência do gânglio ímpar - por pulsos únicos ou múltiplos).

Avaliação do risco de viés: Entre os sete estudos, os estudos de Gonnade et al., ${ }^{17}$ Adas et al., ${ }^{15}$ Sagir et al., ${ }^{6}$ Sencan et al., ${ }^{13}$ Sir e Eksert ${ }^{18}$ e Demircay et al. ${ }^{14}$-foram classificados como "bom," enquanto um estudo de Gopal e McCrory ${ }^{19}$ obteve a classificação "regular," porque não analisou estatisticamente as alterações de pré-para-pós e calculou o valor $p$. Um estudo mencionou a perda de acompanhamento de alguns dos participantes, no entanto, foi menos de $20 \%$ do total de participantes, portanto, não houve rebaixamento da qualidade da avaliação. A avaliação detalhada do risco de viés e a classificação dos estudos incluídos são apresentadas na - Tabela 3.

\section{Resultados}

\section{Características da População}

Foram estudados um total de 189 pacientes (104 no grupo BGI e 85 no grupo ARF). A média de idade variou entre 42,64-42,9 anos no grupo BGI e 42,52-51,3 no grupo ARF. O IMC ponderado foi de $27,24 \mathrm{~kg} / \mathrm{m}^{2}$ e o IMC médio variou entre $24,73-29,49$ $\mathrm{kg} / \mathrm{m}^{2}$ no grupo BGI. O mesmo para o grupo ARF foi de 26,85 $\mathrm{kg} / \mathrm{m}^{2}$ e $26,46-27,98 \mathrm{~kg} / \mathrm{m}^{2}$, respectivamente. Uma história de trauma na região coccígea foi relatado em $64,7 \%$ dos pacientes (33/51) no grupo BGI e 60,6\% dos pacientes (43/71) no grupo ARF. Todos os pacientes do grupo BGI foram tratados por meio de uma abordagem sacrococcígea, enquanto 2 autores preferiram no grupo ARF a abordagem transcoccígea. $\mathrm{O}$ sistema de pontuação mais comumente usado para avaliar a dor foi a EVA ou os homólogos, como EVN, EAN ou EAND. Já que toda a pontuação foi realizada em uma escala de 0 a 10 , elas foram consideradas "semelhantes" para fins de revisão, sendo utilizado um termo comum como "EVA" para representar todas as escalas. A média da pontuação EVA foi de 7,83 no grupo BGI e 6,92 no grupo ARF. 
Tabela 3 Avaliação do RdV nos vários estudos incluídos na revisão

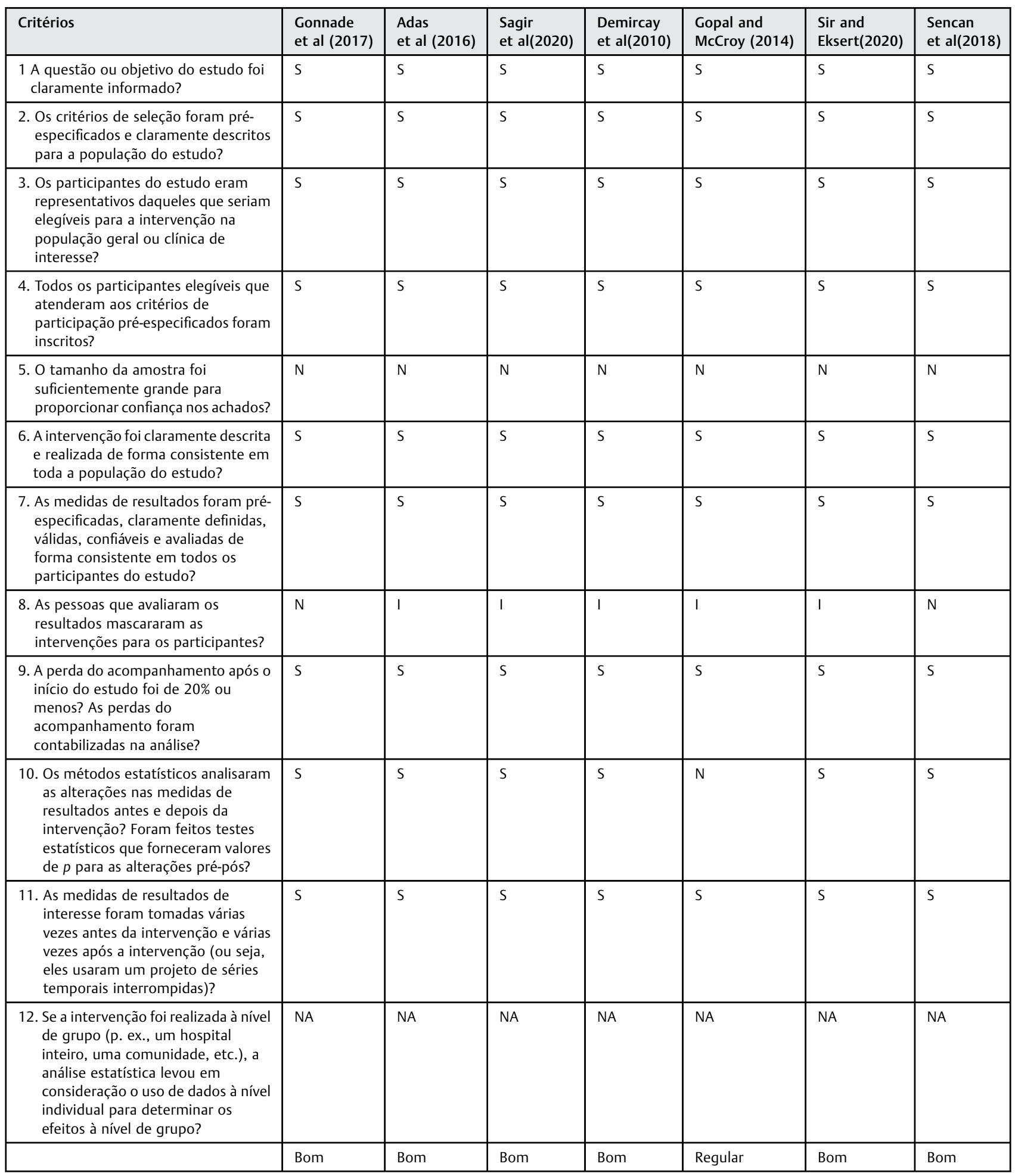

I, INDETERMINADO; N, NÃO; NA, NÃO APLICÁVEL; S, SIM.

\section{Desfecho Primário}

No grupo BGI (-Tabelas 1, 4 e 5), a média EVA foi 7,83 no início do estudo, 3,11 no acompanhamento de curto prazo, 3,55 no acompanhamento de médio prazo e 4,71 no acompanhamento de longo prazo, reduzindo a pontuação da intensidade da dor para $60,28 \%, 54,66 \%$ e 39,85\% no respectivo período de acompa- nhamento, sendo denominado como pontuação \% de alívio, calculado como a diferença entre a EVA basal e a EVA de acompanhamento, expressa como uma porcentagem da EVA basal. No grupo ARF ( - Tabelas 2, 4 e 5), a média da EVA foi de 6,92 no início do estudo, 4,25 no acompanhamento de curto prazo, 4,04 no acompanhamento de longo prazo, resultando em 
Tabela 4 Comparação do desfecho primário das duas modalidades no tratamento da coccigodínia

\begin{tabular}{|c|c|c|c|c|c|c|}
\hline Desfecho Primário & $\begin{array}{l}\text { Bloqueio } \\
\text { Ganglionar }\end{array}$ & $\begin{array}{l}\text { Ablação por } \\
\text { Radiofrequência }\end{array}$ & $\begin{array}{l}\text { Bloqueio } \\
\text { Ganglionar } \\
\text { (pontuação } \\
\text { \% de alívio) }\end{array}$ & $\begin{array}{l}\text { Ablação por } \\
\text { Radiofrequência } \\
\text { (pontuação } \\
\text { \% de alivio) }\end{array}$ & Total & $\begin{array}{l}\text { Total } \\
\text { (\% de alívio) }\end{array}$ \\
\hline EVA- inicial & 7,83 & 6,92 & & & 7,42 & \\
\hline EVA- curto prazo (3-4 semanas) & 3,11 & 4,25 & 60,28 & 38,58 & 3,62 & 51,21 \\
\hline EVA- médio prazo (3 meses) & 3,55 & - & 54,66 & - & - & - \\
\hline EVA- longo prazo (6 meses) & 4,71 & 4,04 & 39,85 & 41,62 & 4,41 & 40,57 \\
\hline Falhas & 11 & 10 & - & - & 21 & - \\
\hline Complicações & 3 & 0 & - & - & 3 & - \\
\hline
\end{tabular}

Tabela 5 Comparação da escala EID (pontuação \% de alívio) nas duas modalidades para coccigodínia no curto e longo prazo

\begin{tabular}{|l|l|l|l|}
\hline Pontuação \% de alívio (EID) & BGI & ARF & Total \\
\hline Curto prazo & 60,28 & 38,58 & 51,21 \\
\hline Longo prazo & 39,85 & 41,62 & 40,57 \\
\hline
\end{tabular}

uma pontuação \% de alívio de $38,58 \%$ e $41,62 \%$ durante o respectivo período de acompanhamento.

\section{Desfecho Secundário}

Foram relatadas no grupo BGI 13,92\% de falhas (11/79) e 2,88\% (3/104) de complicações, enquanto no grupo ARF foram relatadas 14,08\% de falhas (10/71) e nenhuma complicação (0\%). A taxa total de falhas foi de $14 \%$ (21/150) e a taxa de complicações foi de $2,18 \%$ (3/138). Não foram relatadas complicações como infecção ou dor persistente no local da aplicação.

\section{Discussão}

Verificou-se que a coccigodínia ocorre comumente em mulheres. ${ }^{20}$ As pessoas com sobrepeso e obesas são mais propensas a desenvolver coccidínia. ${ }^{2} \mathrm{O}$ IMC médio ponderado da população do estudo foi de $27,04 \mathrm{~kg} / \mathrm{m}^{2}$. A causa mais comum encontrada foi a pós-traumática com $62,29 \%$. Uma lesão traumática pode acontecer de várias maneiras, como a queda de altura, luxação, queda, ATR, traumatismos de parto, bem como microtraumatismos repetidos que passam despercebidos, podendo ser o uso prolongado da bicicleta uma causa para o desenvolvimento da coccidínia.

A irritação crônica das raízes nervosas coccígeas, devido às alterações biomecânicas no cóccix pode ser uma causa de coccidínia. $\mathrm{O}$ gânglio ímpar é o ponto de transmissão nociceptiva coccígea. A irritação crônica do nervo coccígeo torna o gânglio ímpar e o sistema somatossensorial mais sensíveis. ${ }^{21}$ A inibição da transmissão nociceptiva mediante o bloqueio do gânglio ímpar produz um efeito analgésico, diminuindo a sensibilização. ${ }^{13} \mathrm{O}$ êxito do bloqueio depende da localização precisa do gânglio. A localização anatômica do gânglio ímpar continua sendo incerta.

Vários agentes têm sido usados para o bloqueio do gânglio ímpar: anestésicos locais, esteroides, neurolíticos e ablação por radiofrequência. ${ }^{22} \mathrm{O}$ bloqueio ganglionar ímpar com anestésicos locais proporciona um alívio rápido e bom para a coccidínia (dor no cóccix), porém o controle da dor é de curta duração. ${ }^{23} \mathrm{~A}$ extensão do controle da dor pode ser prolongada por meio da neurólise do gânglio.

Na literatura foram descritas várias técnicas de bloqueio ganglionar ímpar (Walther). A injeção guiada por fluoroscopia reduz o risco de complicações como a posição intravascular da agulha, muito anterior (dentro do reto) ou muito superficial (dentro do disco sacrococcígeo). Com o objetivo de aumentar a precisão da aplicação, a localização da ponta da agulha pode ser confirmada com a utilização de contraste antes do procedimento. Plancarte et al. utilizaram uma agulha curva através do ligamento anococcígeo. ${ }^{24} \mathrm{O}$ autor colocou o dedo indicador da mão não dominante no reto para evitar uma ruptura acidental. Wemm e Saberski sugeriram a inserção de uma agulha através do ligamento sacrococcígeo, mediante a técnica transsacrococcígea diretamente no espaço retroperitoneal. ${ }^{25}$ Esta abordagem foi modificada por Munir et al. ${ }^{26}$ para a técnica de agulha dentro da agulha, a fim de evitar o desconforto do paciente, em virtude das múltiplas inserções de agulha, Foye et al descreveram a primeira abordagem da articulação intercoccígea, afirmando que esta abordagem tem a vantagem do injetável estar mais próximo da localização anatômica do gânglio, sendo de fácil visualização na fluoroscopia lateral, em comparação com a articulação SC. ${ }^{27}$ A articulação SC fica obscurecida pelo corno do primeiro cóccix na fluoroscopia lateral. Além disso, a fusão da articulação SC é observada em 52\% dos pacientes com coccidínia idiopática, em comparação com a fusão da articulação intercoccígea, que é observada em apenas $12 \%$ dos casos. Com o objetivo de superar essa dificuldade, Hong et al. ${ }^{28}$ também utilizaram a primeira abordagem da AIC. Toshniwal et al. $^{23}$ descreveram uma técnica alternativa, em caso de calcificação do ligamento sacrococcígeo. Eles inseriram uma agulha através da agulha, por meio de uma agulha introdutora curta e grossa. Alternativamente, a abordagem paramediana foi desenvolvida por Huang et al., ${ }^{29}$ que inseriram uma agulha abaixo do processo transverso do cóccix, redirecionando em direção à linha média. Foye e Patel ${ }^{30}$ utilizaram a abordagem paramediana com a manobra do saca-rolhas.

Além dessas técnicas guiadas por imagem fluoroscópica, outras modalidades de imagem como a TC, USG e a MRI também são usadas para localizar o gânglio. Nesta revisão, todos os estudos utilizaram orientação fluoroscópica e 
material de contraste não iônico, injetado para confirmar a localização exata da ponta da agulha e a propagação do injetável.

Gonnade et al. ${ }^{17}$ obtiveram êxito no BGI com uma única injeção, porém a duração do acompanhamento foi limitada a 6 meses, portanto, foi recomendado um período de acompanhamento mais longo, a fim de avaliar a eficácia do BGI. Ele também obteve respaldo para seus achados com outro sistema de pontuação - Índice de Incapacidade de Oswestry que mostrou uma melhora significativa após um BGI. Juntamente com o BGI, foi adicionada ao regime de tratamento fisioterapia, incluindo exercícios para o assoalho pélvico, para o fortalecimento da virilha e exercícios de alongamento do piriforme para evitar a recorrência. Em um estudo semelhante Sencan et al. ${ }^{13}$ usaram os instrumentos SF-12 para avaliar a qualidade de vida geral e o índice de depressão de Beck, com o intuito de avaliar o estado de humor do paciente. Eles constataram que o SF-12 não mostrou uma melhora significativa nos parâmetros físicos e mentais, nem no curto prazo ( 1 mês) e no longo prazo (6 meses), O IDB mostrou uma melhora significativa, respaldando assim seus resultados e mostrando uma melhora na pontuação EVA com o BGI. A revisão sistemática mostra uma pontuação na EID de $60,28 \%$ em curto prazo e 39,85\% em 6 meses, com uma média de pontuação EVA de 3,11 e 4,71, respectivamente. Esses dados também incluem falhas, portanto, melhorias adicionais na pontuação EVA e EID podem ser esperadas com aqueles que responderam bem ao tratamento, que foi a grande maioria $>85 \%$.

Adas et al. ${ }^{15}$ demonstraram que a RFT transcoccígea é fácil, eficaz e associada a menos complicações. Higuchi et al. ${ }^{31}$ mostraram que a ARF pulsátil para o gânglio dorsal, produz alívio de longo prazo na dor da coluna, sem causar ablação térmica. Gopal e McCroy ${ }^{19}$ não demonstraram nenhum efeito adverso com ARF e uma pontuação EID de 88,88\% aos 6 meses naqueles que responderam a ARF, porém a taxa de falha foi de $25 \%$. Esta foi a pontuação EID máxima relatada por qualquer estudo, tornando a modalidade mais interessante do que o BGI. Demircay et al. ${ }^{14}$ demonstraram melhora significativa na pontuação EVA com ARF transcoccígea, em um número limitado de pacientes, relatando uma pontuação EID de 81,61\% no período pós-procedimento imediato, tendo diminuído gradativamente para 66,67\% em 6 meses de acompanhamento. Além disso, há alguma inconsistência no relato da pontuação EVA, no curto e longo prazo, com uma melhora demonstrada por Gopal e McCroy, ${ }^{19}$ Adas et al. ${ }^{15}$ para ARF e Sagir et al. ${ }^{6}$ para o BGI. Todos os outros estudos mostraram uma redução na EID (aumento na pontuação EVA) no longo prazo, em comparação com o curto prazo. Isso ainda não tem explicação; no entanto, uma razão plausível poderiam ser as variações individuais, resultando na identificação tardia dos não respondentes. Um acompanhamento de mais longo prazo poderia delimitar entre este grupo de pacientes de não respondentes tardios (tendo uma resposta no período inicial de tratamento, porém em última análise não responderam à modalidade de tratamento) e os verdadeiros respondentes (tendo benefícios de longo prazo com as modalidades). Como era previsível, isso é mais comum em pessoas com BGI do que com ARF, pois pode não causar dano permanente ao gânglio da forma mais previsível do que o último. Sagir et al. ${ }^{6}$ também relataram uma diferença significativa em longo prazo nos pacientes tratados com ambas as modalidades e apenas com BGI, resultando em uma pontuação EVA média absoluta de quase metade $(2,4)$ no primeiro, em comparação com o ultimo (5) e um escore EID mais que o dobro (68\%) em comparação com o último (31,83\%). Sir e Eksert $^{18}$ também mostraram uma melhora significativamente melhor na pontuação EVA com ARF do que a modalidade BGI,já que a distribuição do número de pacientes foi bastante desigual em ambos os grupos, com apenas 14 pacientes no grupo ARF, em comparação com 25 no grupo BGI. A partir da - Fig. 2 e das - Tabelas 4 e 5, podemos presumir que o alívio da dor foi maior no grupo BGI no curto prazo, porém, no longo prazo foi numericamente maior no grupo ARF.

Definimos um grupo separado de não respondentes tardios - distinto dos grupos de respondentes e não respondentes, que são facilmente identificados com uma pontuação pós-operatória imediata. Os que não responderam tardiamente são aqueles pacientes que responderam ao tratamento no estágio imediato (indicado por uma diminuição na pontuação EVA), porém apresentaram uma tendência a atingir os níveis de EVA pré-procedimento, em médio e longo prazo (3 meses após o procedimento). 0 motivo da existência desses pacientes pode ser a presença de um limiar mais alto para o bloqueio ou ablação, ou ainda a colocação inadequada da agulha ou sonda. Além disso, recomendamos o uso da EID (pontuação \% de melhora) para quantificar a redução na pontuação EVA. Em vez de uma redução absoluta, a escala EID deve ser mais claramente correlacionada com a melhora da qualidade de vida embora esta seja uma afirmação inicial e precise de validação através de estudos futuros. Porém, teoricamente falando, a quantidade de satisfação mental e o alívio físico não pode ser igual quando, digamos, estamos falando de uma redução absoluta de 4 na pontuação EVA - será diferente de uma redução de 6 para 2 do que de 9 para 5 , indicando uma pontuação na EID de $66,67 \%$ no primeiro e $44,44 \%$ no último.

O ponto forte do presente estudo de revisão é que todos os estudos incluídos tiveram uma qualidade de evidência classificada como bom ou regular, determinada pelo uso das ferramentas do NIH. O efeito da intervenção foi observado para a duração de longo prazo (6 meses) em todos os estudos. Também há algumas limitações, pois nenhum ensaio comparativo randomizado estava disponível, portanto, não foi

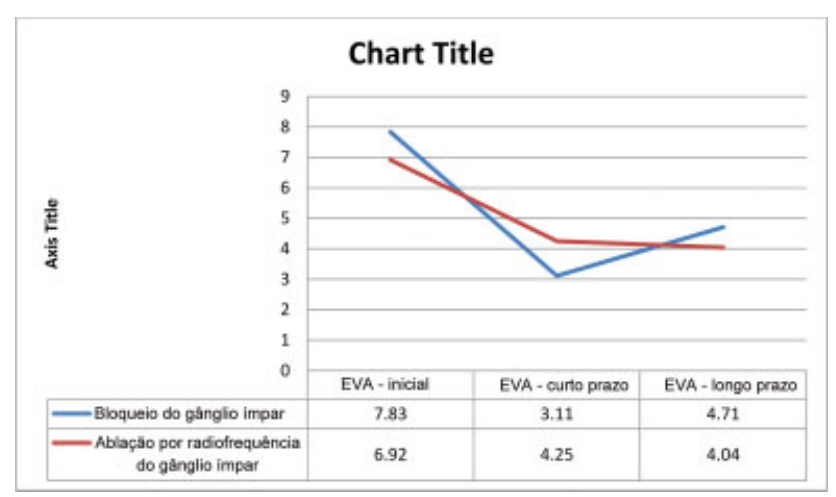

Fig. 2 Diagrama de linha, representando a queda e o aumento do escore EVA nos dois grupos (BGl e ARF) após o tratamento para coccidínia. 
incluído na revisão, já que alguns deles não relataram parâmetros demográficos básicos, não sendo realizada uma revisão detalhada com base nos dados demográficos. Além disso, os pacientes com coccigodínia de etiologia traumática, idiopática e maligna não são avaliados separadamente, devido à indisponibilidade de dados separados. Mais uma vez, a radiofrequência contínua e pulsátil foi considerada igual na categoria de ARF. Certamente existe uma necessidade de grandes estudos comparativos e randomizados sobre o bloqueio ganglionar ímpar, com o uso de esteroides e neuromodulação por radiofrequência. No entanto, olhando para os resultados dos estudos publicados, não seria insensato afirmar que, independentemente da modalidade utilizada, elas proporcionam um excelente alívio da dor na maioria dos pacientes.

\section{Conclusão}

O BGI e ARF são opções intermediárias de tratamento, entre uma opção conservadora de tratamento clínico e uma opção radical de excisão cirúrgica. Elas são minimamente invasivas, podendo eliminar um custo cirúrgico desnecessário para a maioria dos pacientes que não respondem ao tratamento clínico conservador. Considerando uma taxa de desfecho bem sucedida de $>85 \%$ com qualquer modalidade e quase $90 \%$ com os meios conservadores, a necessidade de excisão cirúrgica é reduzida para $<1,5 \%$ dos pacientes com coccigodínia. Os autores recomendam um ensaio com BGI ou ARF nos pacientes que não respondem ao tratamento clínico conservador, juntamente com a fisioterapia. A escolha do BGI ou ARF dependerá da disponibilidade de recursos, da habilidade do médico assistente e da escolha do paciente.

Conflito de Interesses

Os autores declaram não haver conflito de interesses.

\section{Referências}

1 Simpson J. Coccygodynia and diseases and deformities of the coccyx. Med Times Gaz 1859;40(01):1-7

2 Maigne JY, Doursounian L, Chatellier G. Causes and mechanisms of common coccydynia: role of body mass index and coccygeal trauma. Spine 2000;25(23):3072-3079

3 Pennekamp PH, Kraft CN, Stütz A, Wallny T, Schmitt O, Diedrich O. Coccygectomy for coccygodynia: does pathogenesis matter? J Trauma 2005;59(06):1414-1419

$4 \mathrm{Kim} \mathrm{NH}$, Suk KS. Clinical and radiological differences between traumatic and idiopathic coccygodynia. Yonsei Med J 1999;40 (03):215-220

5 Maigne JY, Pigeau I, Aguer N, Doursounian L, Chatellier G. Chronic coccydynia in adolescents. A series of 53 patients. Eur J Phys Rehabil Med 2011;47(02):245-251

6 Sagir O, Demir HF, Ugun F, Atik B. Retrospective evaluation of pain in patients with coccydynia who underwent impar ganglion block. BMC Anesthesiol 2020;20(01):110

7 Capar B, Akpinar N, Kutluay E, Müjde S, Turan A. [Coccygectomy in patients with coccydynia]. Acta Orthop Traumatol Turc 2007;41 (04):277-280

8 Trollegaard AM, Aarby NS, Hellberg S. Coccygectomy: an effective treatment option for chronic coccydynia: retrospective results in 41 consecutive patients. J Bone Joint Surg Br 2010;92(02):242-245

9 Howard PD, Dolan AN, Falco AN, Holland BM, Wilkinson CF, Zink AM. A comparison of conservative interventions and their effec- tiveness for coccydynia: a systematic review. J Manual Manip Ther 2013;21(04):213-219

10 Karadimas EJ, Trypsiannis G, Giannoudis PV. Surgical treatment of coccygodynia: an analytic review of the literature. Eur Spine J 2011;20(05):698-705

11 Lim SJ, Park HJ, Lee SH, Moon DE. Ganglion impar block with botulinum toxin type a for chronic perineal pain -a case report-. Korean J Pain 2010;23(01):65-69

12 Oh CS, Chung IH, Ji HJ, Yoon DM. Clinical implications of topographic anatomy on the ganglion impar. Anesthesiology 2004;101 (01):249-250

13 Sencan S, Kenis-Coskun O, Demir FGU, Cuce I, Ercalık T, Gunduz OH. Ganglion Impar block improves neuropathic pain in coccygodynia: A preliminary report. Neurol Neurochir Pol 2018;52(05):612-617

14 Demircay E, Kabatas S, Cansever T, Yilmaz C, Tuncay C, Altinors N. Radiofrequency thermocoagulation of ganglion impar in the management of coccydynia: preliminary results. Turk Neurosurg 2010;20(03):328-333

15 Adas C, Ozdemir U, Toman H, Luleci N, Luleci E, Adas H. Transsacrococcygeal approach to ganglion impar: radiofrequency application for the treatment of chronic intractable coccydynia. J Pain Res 2016;9:1173-1177

16 Bijur PE, Latimer CT, Gallagher EJ. Validation of a verbally administered numerical rating scale of acute pain for use in the emergency department. Acad Emerg Med 2003;10(04):390-392

17 Gonnade N, Mehta N, Khera PS, Kumar D, Rajagopal R, Sharma PK. Ganglion impar block in patients with chronic coccydynia. Indian J Radiol Imaging 2017;27(03):324-328

18 Sir E, Eksert S. Comparison of block and pulsed radiofrequency of the ganglion impar in coccygodynia. Turk J Med Sci 2019;49(05): 1555-1559

19 Gopal H, Mc Crory C. Coccygodynia treated by pulsed radio frequency treatment to the Ganglion of Impar: a case series. J Back Musculoskeletal Rehabil 2014;27(03):349-354

20 Nathan ST, Fisher BE, Roberts CS. Coccydynia: a review of pathoanatomy, aetiology, treatment and outcome. J Bone Joint Surg Br 2010;92(12):1622-1627

21 De Andrés J, Chaves S. Coccygodynia: a proposal for an algorithm for treatment. J Pain 2003;4(05):257-266

22 Reig E, Abejón D, del Pozo C, Insausti J, Contreras R. Thermocoagulation of the ganglion impar or ganglion of Walther: description of a modified approach. Preliminary results in chronic, nononcological pain. Pain Pract 2005;5(02):103-110

23 Toshniwal GR, Dureja GP, Prashanth SM. Transsacrococcygeal approach to ganglion impar block for management of chronic perineal pain: a prospective observational study. Pain Physician 2007;10(05):661-666

24 Plancarte R, Amescua C, Patt RB, Allende S. A751 presacral blockade of the ganglion of walther (Ganglion impar). [abstract]. Anesthesiol J Am Soc Anesthesiol 1990;73(3A):A751

25 Wemm K Jr, Saberski L. Modified approach to block the ganglion impar (ganglion of Walther). Reg Anesth 1995;20(06):544-545

26 Munir MA, Zhang J, Ahmad M. A modified needle-inside-needle technique for the ganglion impar block. Can J Anaesth 2004;51 (09):915-917

27 Foye PM. Ganglion impar blocks for chronic pelvic and coccyx pain. Pain Physician 2007;10(06):780-781

28 Hong JH, Jang HS. Block of the ganglion impar using a coccygeal joint approach. Reg Anesth Pain Med 2006;31(06):583-584

29 Huang JJ. Another modified approach to the ganglion of Walther block (ganglion of impar). J Clin Anesth 2003;15(04):282-283

30 Foye PM, Patel SI. Paracoccygeal corkscrew approach to ganglion impar injections for tailbone pain. Pain Pract 2009;9(04): 317-321

31 Higuchi Y, Nashold BS Jr, Sluijter M, Cosman E, Pearlstein RD. Exposure of the dorsal root ganglion in rats to pulsed radiofrequency currents activates dorsal horn lamina I and II neurons. Neurosurgery 2002;50(04):850-855 